\title{
Astronomy in the 1980s
}

\author{
Astrophysics and geophysics are ESA's two oldest disciplines. Professor A. P. Willmore, of the \\ University of Birmingham's Space Research Department presents the case for launching \\ sophisticated equipment to do high energy astronomy ...
}

EVEn more than for most sciences, the last two decades in astronomy have been a period of rapid technological development for all its branches. The substitution of high quantum-efficiency detectors for the photographic plate in optical telescopes, the application of large aperture synthesis telescopes in radio astronomy and the entire histories of infra-red, ultra-violet, X-ray and $\gamma$-ray astronomies are contained within the last 20 years. The application of spaceflight to astronomy, on which the possibility of the newest branches of astronomy (only excluding work in the infrared) has depended and which promises some important advances in the older branches too, has also occurred during this time.

The last two decades have also been a quite exceptional period of discovery whose many results now demand exploitation. It is also most unlikely that the phases of technological development and discovery are yet at an end. Thus charting a course for astronomy, especially space astronomy which is so costly, will require careful selection from a broad spectrum of projects, any of which in less exciting times might have seemed suitable to be pursued.

Space astronomy-for the obvious reason again of its expense-is highly internationalised; most experiments or facilities involving international teams with members from both sides of the Atlantic. The degree of internationalisation exceeds that even in nuclear physics so that a distinctively European programme is hard to conceive and it is useful only on a world scale to identify the promising paths to develop. But several branches of astronomy are alive and well in Europe, particularly in the UK, and there is no reason to doubt that UK astronomers will be well represented in world class astronomy, provided of course that funding at a minimum necessary level is available.

The sensitivity of large optical telescopes has been substantially enhanced in recent years by the development of photo-electric detectors whose quantum efficiency is far higher than that of photographic plates and whose performance in other respects is comparable. However, the ability of a telescope to detect and resolve objects which are very distant and therefore faint and of small angular diameter depends also on its effective image quality and on the brightness of the night sky. The angular resolution of any good, large optical telescope is determined not by diffraction or the quality of its construction but by the atmosphere, and consequently the Space Telescope, a $2,4 \mathrm{~m}$. orbiting telescope being built by NASA with ESA as a minor partner, will have an effective resolution 10 times and a sensitivity 100 times that of the large ground-based telescopes

The Space Telescope can operate over a wide wavelength range, and will be an ideal instrument for the study of the most distant galaxies which are of course those which are observed in the earliest epochs in the Universe, closest to the time of the Big Bang, and around the epoch of galaxy formation. The measurements of the motions and parallaxes (the apparent motions due to the Earth's orbital motion about the Sun, on which ultimately all astronomical distance scales depend) and of astronomical objects are severely limited by atmospheric effects, and either with the Space Telescope or with satellites constructed for the purpose, improvements of about an order of magnitude should be attainable.

The short history of X-ray astronomy is especially remark- able for the profusion of new discoveries, for example the close binary sources containing compact stars such as neutron stars and-one supposes-black holes, which allow properties-the mass, at least-of the compact star to be determined in a number of cases. It is salutary to recognise the relative crudity of the techniques which have generally been employed in making these observations, which are about equivalent to carrying out optical astronomy with something between a photographic light meter and a box camera. But much technique development has been carried out in the last 10 years, and the new techniques are now ready to be exploited. Later this year the NASA HEAO-B satellite should be launched with a medium size grazing incidence telescope. This will have an angular resolution roughly two orders higher than any previous $\mathrm{X}$-ray instrument, comparable for the first time with that of an optical telescope. The sensitivity too will be far higher than that of any previous instrument-indeed it will be much better than that of any present X-ray sky survey, which will undoubtedly prove some limitation on its use. This telescope is likely to be able to detect the X-ray emission from clusters of galaxies at distances approaching those of the most distant quasars, thus providing another means of observing the early Universe. Other means of imaging at higher X-ray energies have also been developed, and this is a whole field that is sure to prove productive.

Recent observations of X-ray spectra, which like the spectra of radio sources are dominated by continuum emission, have shown the presence of measurable line emission in a wide variety of sources. This generally arises from highly ionised atoms, usually of $\mathrm{Fe}$, which are present in the source, which must be at a high temperature since it is an X-ray emitter. A high energy feature, however, is believed to be cyclotron emission from around a neutron star, thus allowing for the first time a direct measurement of the large magnetic field (about $10^{8} \mathrm{~T}$ ) around these objects. The diagnostic value of such detailed spectrum observations is very high and together with much improved observations of the spatial structure of sources this is bound to prove an area of important development.

$X$-ray observations are generally considered to extend up to the electron-positron annihilation radiation at $512 \mathrm{keV}$. Observations of the $\gamma$-ray sky at higher energies are relatively scanty, depending on the surveys carried out with spark chambers on the SAS-2 and Cos-B satellites. Whilst X-ray and optical objects such as the Crab nebula and the Vela pulsar are detected at $\gamma$-ray energies, there is a good chance that the typical $\gamma$-ray source will prove to be qualitatively different from those observed at lower energies and promising new observational techniques such as drift chambers and double Compton scatter telescopes are now in existence.

In selecting the projects of the future, the obvious criteria to use (and one implicitly accepted in the examples already cited) will be the extent to which they are likely to contribute to fundamental topics-either cosmology, or fundamental physics as general relativity or the properties of nuclear matter. But throughout high energy astronomy, better surveys will be vitally important in the next decade, and will surely bring to light many new types of astronomical object. It will be worth remembering that even at lower energies, both pulsars and quasars were discovered within the last 20 years or so. 